\title{
Genetic Based Time Synchronization Optimize Algorithm for Wireless Sensor Networks
}

\author{
Chen Chang ${ }^{1,2}$ \\ $3^{\text {rd }}$ Department \\ Beijing Aerospace Automatic Control Institute ${ }^{1}$ \\ National Key Laboratory of Science and Technology \\ on Aerospace Intelligence Control ${ }^{2}$ \\ Beijing, China \\ changchen@bit.edu.cn \\ Wang Dongxiao ${ }^{3}$ \\ School of Optoelectronics \\ Beijing Institute of Technology ${ }^{3}$ \\ Beijing, China \\ wangdongxiao@bit.edu.cn
}

\author{
Xiao Liangang ${ }^{1}$ \\ $3^{\text {rd }}$ Department \\ Beijing Aerospace Automatic Control Institute ${ }^{1}$ \\ Beijing, China
}

\begin{abstract}
Time synchronization provides uniform timescale for distributed sensor networks. In this paper a genetic based time synchronization optimize algorithm is proposed. It first generated the nondeterministic transmitting delay and then adopted the genetic algorithm to simulating these delays. Minimal linear fit residual error is selected as fitness function to evaluate the simulating effect. After finding the best fitness delay sequence, the time skew and offset is estimated by linear regression. The experiment result shows that the synchronization precision could be improved from $11.8^{\mu s}$ to $3.5^{\mu s}$.
\end{abstract}

Keywords-Sensor networks; Time synchronization;Genetic algorithm;Optimize;Precision

\section{INTRODUCTION}

Time synchronization provides uniform timescale for distributed sensor network. It is required for several applications including coordinating events in decentralized system[1] and data fusion[2]. More broadly, as we head towards the era of event-cum-time-driven systems featuring the convergence of computation and communication with control, the need for well synchronized clocks becomes increasingly important [3].

Most of the time, synchronization algorithm is a tradeoff between precision and consumption. In the one hand, an ideal algorithm should achieve higher synchronization precision under determinate power consumption. In the other hand, it should consume less resource to achieve a determinate synchronization precision.

Generally speaking, high precision time synchronization means it could not only calibrate the time offset, but also could estimate the time skew between two crystal oscillators. Linear regression or least square estimation is a widely used method, which is equivalent to MLE in some conditions. RBS [5] and FTSP [4] adopted this estimator to estimate the clock offset and skew. Jeff S. Pettyjohn, et. [6] proposed a least squares based estimation of relative clock offset and frequency for high latency sensor networks. The research result shows that the combination of a feasible checked least square estimator with the Paxsion-base estimator performs comparable and sometimes better than the constrained least square based estimator. Moreover, Aggelos Bletsas [7] proposed a Kalman Filter algorithm to estimate the clock offset and skew in sender-receiver message mechanism, which is significance to time parameters estimation. It could benefit by scheduled transmission from the client system that ensures minimum delay variance due to the operating system. However, all of these methods could not get a "better" result if the non-deterministic delay is larger.

For the purpose of getting a higher time synchronization precision with the condition of larger nondeterministic delay or more broadly, decreasing the communication consumption by sacrifice computing consumption, a genetic based time synchronization optimize algorithm is proposed. It pays more attention on how to get a more precise time skew and offset estimation, but not the message exchanging mechanism. More detailed, it first gets a sequence of original time drift which contains actual time drift and non-deterministic message transmitting delay. After that it generates a sequence of random number to simulate the non-deterministic delay. Original time drift minus these random numbers is actual time drift. And then genetic algorithm is used to optimize these random numbers. The linear regression coefficient which could get smallest residual error is considered as best fitness. As compared with traditional linear regression method, the new method could get a synchronization precision of 3 us.

The remainder of this paper is organized as follows. In section 2 the mathematic model of time synchronization is described. After that the genetic based time synchronization optimize method is proposed in section 3 . In section 4 the optimize result is evaluated. Finally we discussed the conclusion in section 5 .

\section{MATHEMATIC MODELS OF CLOCK}

Without considering the message exchanging mechanism, the optimize algorithm is founded on the 
clock model, which is determined by the physical property of oscillator.

The underlying reason of time drift is the physical inconsistence of crystal oscillator, which could be formulated by clock model. As it is discussed in [8], the clock model could be described by linear model, which include affine mappings. Assume the universal time coordinate (UTC) is denoted by $\mathrm{t}$, which is a fixed reference clock. According to the linear model, the time of node $\mathrm{i}$ could be described as

$$
T_{i}(t)=a_{i} t+b_{i}
$$

Where $a_{i}$ named clock skew, $b_{i}$ named clock offset.

Suppose there are two nodes A and B, which time could be represented by $T_{A}(t)$ and $T_{B}(t)$, respectively. The time offset of node A and B is denoted as $\phi_{A B}(t)=T_{A}(t)-T_{B}(t)$, and the time skew of node A and $\mathrm{B}$ is denoted as[9][10].

$$
\theta_{A B}(t)=\frac{d C_{A}(t)}{d t}-\frac{d C_{B}(t)}{d t}
$$

The time offset, which arose from the difference of initial power-on time, could be eliminated. While the time skew, which arose from the physical property difference of oscillators, could only be corrected.

Considering node $\mathrm{A}$ as the reference clock, the relative time offset and time skew of node $\mathrm{B}$ could be represented by

$$
T_{\text {drift }}(t)=\theta_{A B} t+\phi_{A B} \text {. }
$$

Where $\theta_{A B}=\frac{\theta_{B}}{\theta_{A}}, \phi_{A B}=\phi_{B}-\frac{\theta_{B}}{\theta_{A}} \phi_{A}$.

The relationship of time offset and skew is shown in Figure.1. The offset at $t_{0}$ is the initial time drift. The dash line represents the relationship of $\left\{t, T_{A B}(t)\right\}$.

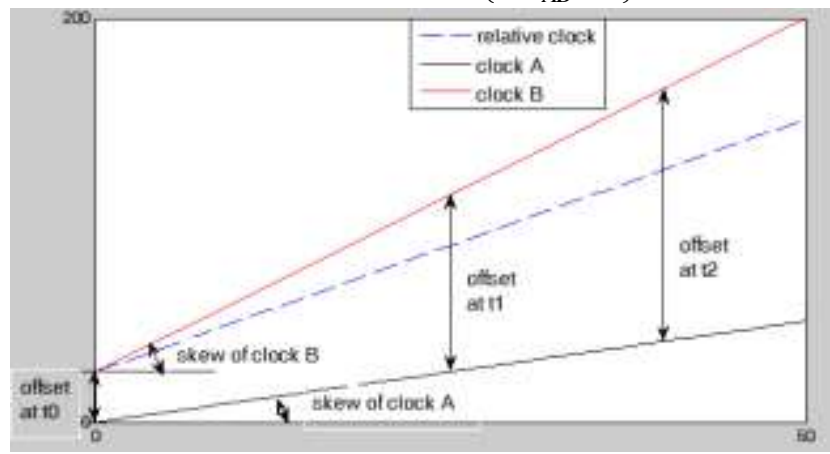

Figure 1. relationship of time offset and skew

Unfortunately, this relationship is more or less drowned by non-deterministic time delay, which is arose by timestamp message exchanging. Considering the nondeterministic delay as disturbance, it is possible to eliminate the disturbance of $T_{d r i f t}$ by mathematic method.

\section{GENETIC BASED TIME SYNCHRONIZATION OPTIMIZE ALGORITHM}

As it is illustrated in Figure.2, the process of genetic optimize algorithm is consists as three modules: original time drift get module, disturbance initialize module and genetic optimize module.

\section{A. Original time drift get module}

Suppose there are two nodes A and B, which time could be represented by $T_{A}(t)$ and $T_{B}(t)$. Set Node A as reference node, that is, node $B$ sends it's timestamp message to A. After $\mathrm{N}$ times timestamps exchanging, node A could collect a sequence of timestamps $\left\{T_{A}(i), T_{B}(i)\right\}(i=1,2, \ldots . N)$. The relative time drift $T_{A B}(i)$ could be represented as follows:

$$
T_{A B}(i)=T_{A}(i)-T_{B}(i)
$$

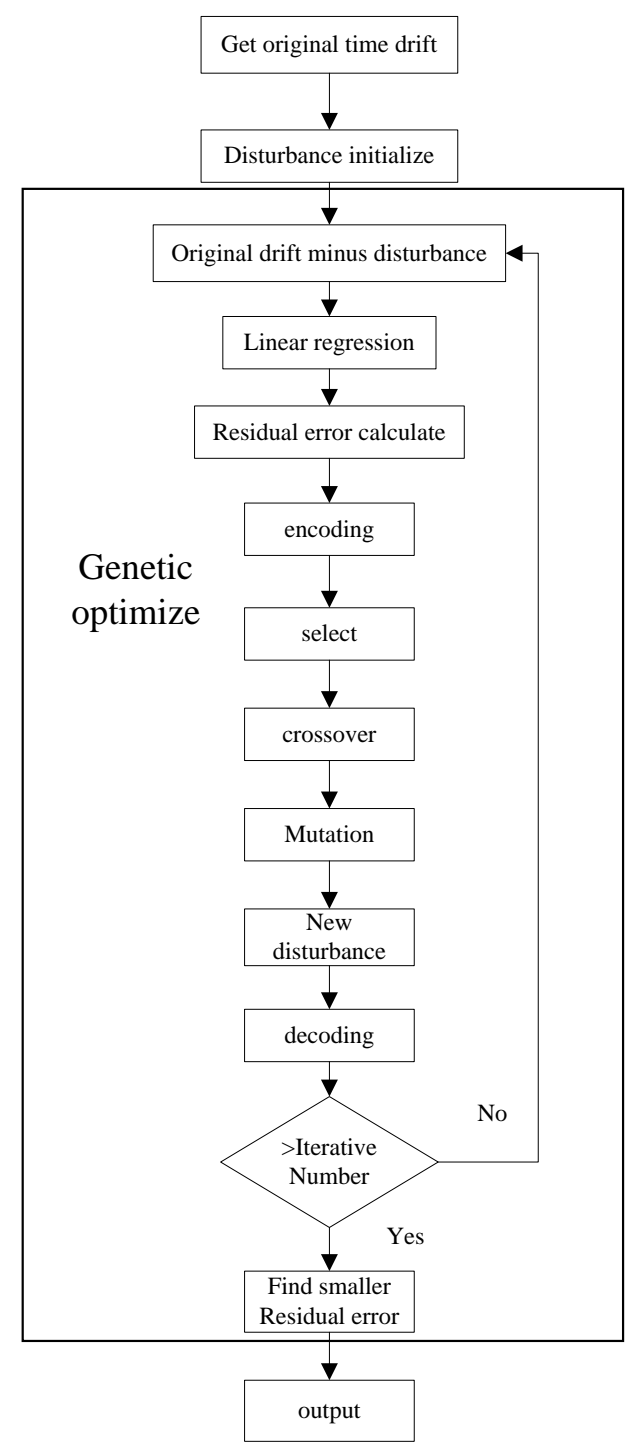

Figure 2. genetic optimize algorithm framework

\section{B. Disturbance initialize module}

Considering the clock model and the non-deterministic delay during message exchanging, (2) could be rewrite as

$T_{A B}(i)=\theta_{A B} * i+\phi_{A B}+w(i)$

$w(i)$ is the non-deterministic transmitting delay. 
Suppose $w(n)$ is a white Gaussian noise with mean zero and variance $\sigma^{2}$, the Joint probability density of $\{(\gamma(i)) \mid i=1,2, \ldots . . n\}$ is

$$
\begin{aligned}
& L=\prod_{i=1}^{n} \frac{1}{\sigma \sqrt{2}} \exp \left[-\frac{1}{2 \sigma^{2}}(\gamma(i)-\phi-\theta * i)^{2}\right] \\
& =\left(\frac{1}{\sigma \sqrt{2}}\right)^{n} \exp \left[-\frac{1}{2 \sigma^{2}} \sum_{i=1}^{n}(\gamma(i)-\phi-\theta * i)^{2}\right]
\end{aligned}
$$

It is obvious that

$$
\max (L)=\left(\frac{1}{\sigma \sqrt{2}}\right)^{n} \exp \left[-\frac{1}{2 \sigma^{2}} \min \left(\sum_{i=1}^{n}\left(\gamma(i)-\phi-\theta^{*} i\right)^{2}\right)\right]
$$

Here the problem changes into classical Ordinary Least Squares estimate [11].

It is possible to generate a sequence of random number to simulate this stochastic process. If the simulation of this stochastic process is exactly enough, the linear regression estimation result could close enough to the actual clock model. The purpose of genetic algorithm is to get an exactly enough simulation result.

\section{Genetic optimize algorithm}

Genetic algorithm is a global probability search algorithm based on genetic heredity mechanism. It first changed the search parameters into binary coding, and then adopted selection, random crossover and mutation operation to generate new binary coding sequence. Fitness function is adopted to evaluate the evolving result.

Therefore, the basic element of genetic algorithm is list as follows:

Destination function mathematic models: clock and disturbance models, which is illustrated by(2).

Coding strategy: set random sequence numbers as search parameters and changing them into traditional binary coding.

fitness function: linear regression residual error of eq.2. Generally speaking, the random sequence number which is close enough to actual non-deterministic could get smaller enough linear regression residual error.

heredity strategy: set population size as 20 , evolving generation is 500, crossover probability is 0.9 , and mutation probability is 0.05 . Crossover and mutation strategy is according to basic genetic algorithm.

According to Figure.2, after 500 generations evolving, we could find a sequence of random number which generates smallest linear regression residual error. However, neither this sequence of random number and residual error is our aim. According the clock model, the linear regression coefficient is our final destination. That is the sequence of random number which generates smallest linear regression residual error is closer to actual nondeterministic timestamp transmitting delay. Based on this number sequence, we could get a more exactly clock model coefficients.

\section{OPTIMIZE RESULT}

In this section we evaluated this algorithm by experiment.
The original time drift that collected by unidirectional message exchanging mechanism is shown in Figure.3. The symbol "*" shows the original data, and the red line is the linear regression fit line. The fit equation is $t$ (i) $=3609.2$ $4.375 * \mathrm{i}$. that is, the result of clock offset is $3609.2 \mu \mathrm{s}$, clock skew is $-4.375 \mu s$. Combined with the synchronization periodic of 2 second, we could get the clock skew is $-4.375 / 2=-2.1875 \mu s$.The residual error statistic result is shown in Figure. 4

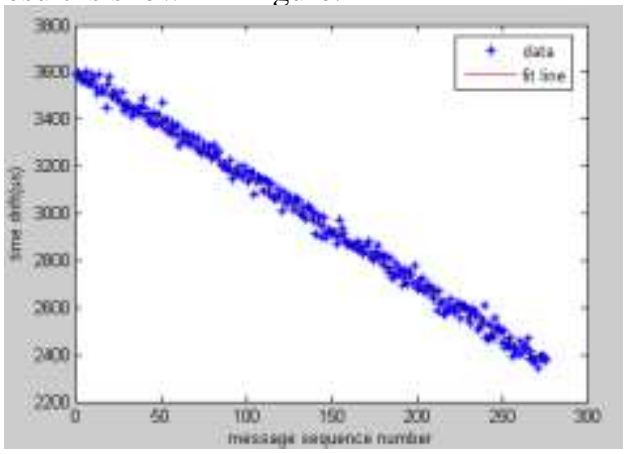

Figure 3. Original time drift

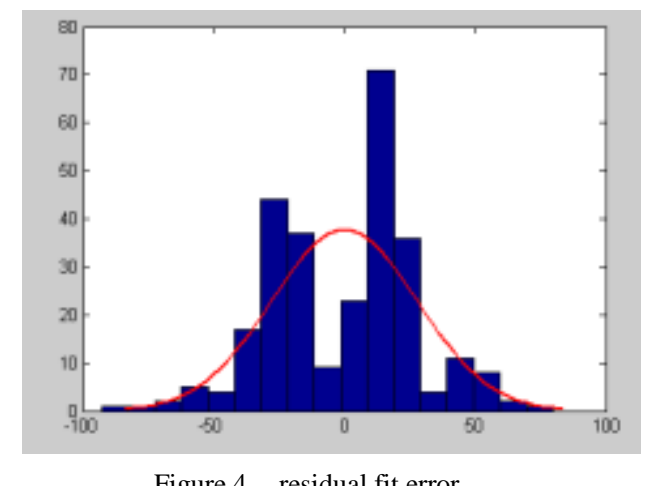

The actual time drift is shown in Figure.5. We use the oscilloscope to observe the difference of GPIO pin's signal rising time to determine the synchronization error. The observation started at 23:07:30, ended at 23:37:15.Every 3 minutes we record the time difference. The initial time drift and clock skew could be easily got by linear regression, which is $3619 \mu \mathrm{s}$ and $-2.4425 \mu \mathrm{s} / \mathrm{s}$.

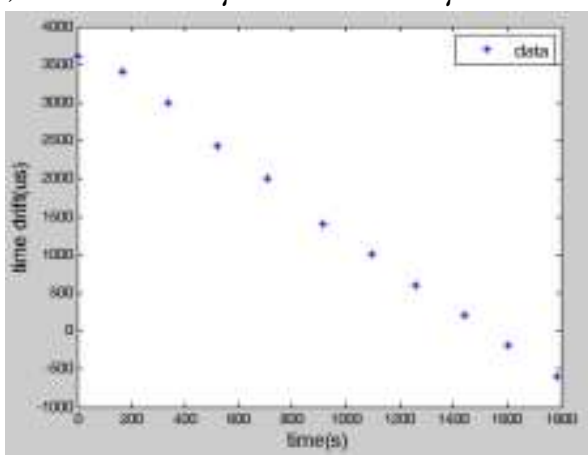

Figure 5. observed data

Here we could get the synchronization error. The initial clock offset error is (3619-3607.2) $\mu s=11.8 \mu \mathrm{s}$. The clock skew error is $(-2.4425+2.1875) \mu s=-0.225 \mu s$. 
As comparison, the genetic based time synchronization optimize result is shown as follows.

After 500 generations optimize, the fit result is $\mathrm{t}$ (i) $=3615.5-4.525 * \mathrm{i}$. That is, the initial clock offset error is (3619-3615.5) $\mu s=3.5 \mu \mathrm{s}$. The clock skew error is ($2.4425+2.2625) \mu s=-0.18 \mu s$.

The residual error statistic result is shown in Figure.6. Compared with figure.4, it could be seen that the fit residual error distributing is more convergent and the residual error is smaller.

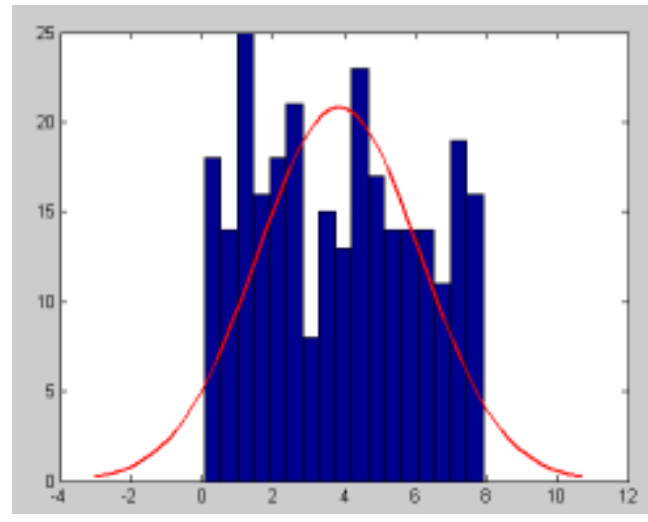

Figure 6. residual fit error

Compared with traditional linear regression method, it could be seen that the synchronization precision improved $8.3 \mu \mathrm{s}$ and the skew estimation precision improved $0.0425 \mu s / \mathrm{s}$. That is, the synchronization precision improved more than $70 \%$. On the contrary, for the purpose of maintaining the same synchronization precision for sensor networks, the optimize algorithm saves more than $70 \%$ communication consumption while increasing more compute consumption.Considering the power consumption diversity between communication and compute, the optimize algorithm is benefit for decreasing sensor networks resource.

After that, the stabilization of this optimize algorithm is evaluated. The algorithm is executed for 10 times, and the result is shown in Table.1.

TABLE I. 10 TimES OPTIMEIZ RESUlt

\begin{tabular}{|l|l|l|}
\hline & Offset $(\mu s)$ & Skew $(\mu s / \mathrm{s})$ \\
\hline 1 & 3615.5 & -4.525 \\
\hline 2 & 3615.5 & -4.526 \\
\hline 3 & 3615.1 & -4.5252 \\
\hline 4 & 3615.6 & -4.526 \\
\hline 5 & 3615.5 & -4.5265 \\
\hline 6 & 3615.9 & -4.5285 \\
\hline 7 & 3615.3 & -4.5237 \\
\hline 8 & 3615.4 & -4.5253 \\
\hline 9 & 3615.8 & -4.5284 \\
\hline 10 & 3615.7 & -4.5269 \\
\hline
\end{tabular}

The time offset estimate variance is less than $1 \mu \mathrm{s}$. And the time skew estimate variance is less than $0.01 \mu \mathrm{s}$.

It could be seen that the optimize result is nearly the same, which means the algorithm is stabilize enough.

\section{CONCLUSION}

In this paper a genetic based time synchronization optimize algorithm is proposed and evaluated. After generate and simulate the nondeterministic message transmitting delay by genetic algorithm, the time skew and offset between two nodes could be estimated by linear regression. The experiment result shows that the algorithm could efficiently estimate the time skew and offset. Compared with non-optimized linear regression method, the synchronization precision could improve $70 \%$, that is, the synchronization precision improved from $11.8 \mu \mathrm{s}$ to $3.5 \mu s$.

\section{ACKNOWLEDGMENT}

The acknowledgment is for our colleagues. Thank you for your cooperation in complying with these instructions.

\section{REFERENCES}

[1] S.Graham and P.R.Kumar. The convergence of control, communication, and computation[C]. Proc. PWC 2003,Vol.2775, Berlin,Germany: Springer-Verlag, 2003, p.458-475.

[2] C.Lin,W.Peng and Y.Tseng. Efficient in-network moving object tracking in wireless sensor networks[J]. IEEE Trans.Mobile Comput.,Vol. 5(8), p.1044-1056, Aug.2006.

[3] Nikolaos M.Freris, Hemant Kowshik, P.R.Kumar. Fundamentals of large sensor networks: connectivity, capacity, clocks, and computation. [C].Proceedings of the IEEE, Vol.98(11), p.18281846,Nov.2010

[4] Miklos Maroti, Branislav Kusy, Gyula Simon, and Akos Ledeczi. The flooding time synchronization protocol[C]. SenSys'04. November 3-5, 2004. Baltimore,USA. P.39-49.

[5] Elson,J.E., Girod,L., and Estrin,D. Fine-Grained Network time synchronization using reference broadcasts[C]. The Fifth Symposium on Operating System Design and Implementation (OSDI), p.147-162,December 2002.

[6] Jeff S.Pettyjohn, Daniel R.Jeske and Jun Li. Least squares based estimation of relative clock offset and frequency in sensor networks with high latency $[\mathrm{J}]$. IEEE transaction on communications, Vol.58(12). p.3613-3620. Dec. 2010.

[7] Aggelos Bletsas. Evaluation of kalman filtering for network time keeping[J]. IEEE transactions on ultrasonics, ferroelectrics, and frequency control, Vol.52(9). September 2005.

[8] N.Fferis, S.Graham, and P.R.Kumar. Fundamental limits on synchronization clocks over networks[J]. IEEE Trans.Autom. Control. Vol.56(6).P.1352-1364.June.2011.

[9] Undararamnan B.,Buy U.,Kshemkalyani A.D. Clock synchronization in wireless sensor networks: A Survey[C]. Ad-Hoc Networks, 2005.Vol.3(3):281-323.

[10] Ganeriwal S.,Capkun S.,Han C., and Srivastava M.B. Secure time synchronization service for sensor networks. Proceeding of the 4th ACM workshop on wireless security (WISE'05) [C]. Germany: Cologne. 2005:97-106.

[11] D. C Montgomery, E. A. Peck, and G. G. Vining, Introduction to Linear Regression Analysis, 3rd edition[M]. New York: WileyInterscience, 2001. 